\title{
Contribution of the rib cage to breathing in tetraplegia
}

\author{
MDL MORGAN, AR GOURLAY, JR SILVER, SJ WILLIAMS, DM DENISON \\ From the Lung Function Department, Brompton Hospital, London; UK Scientific Centre, IBM, Winchester; \\ and the National Spinal Injuries Centre, Stoke Mandeville
}

ABSTRACT In tetraplegia there is often paradoxical inward motion of the rib cage during inspiration. The volume of this negative contribution is difficult to estimate but can be obtained by optical mapping. The partitioning of ventilation between the rib cage and abdomen in six normal subjects, 10 stable tetraplegic patients, and one tetraplegic patient at intervals during rehabilitation has been studied by this technique. In normal subjects the tidal volume and the vital capacity were the sum of positive contributions from the rib cage and abdomen. In stable tetraplegic subjects with similar neurological levels, the rib cage contribution varied widely but the total chest wall displacement could not be predicted from the vital capacity. In the patient studied sequentially rib cage paradox reversed with time after injury, and this was associated with an absolute increase in vital capacity and an improvement in the action of the diaphragm.

Several authors have drawn attention to the functional importance of paradoxical motion of the rib cage in tetraplegia..$^{-4}$ This is believed to account in part for the very low vital capacity that may be seen after injury. A reduction in paradoxical motion is often associated with an improvement in vital capacity that occurs with time, even in the apparent absence of neuromuscular recovery. It has not previously been possible to make a volumetric estimate of this motion. This paper describes a method of doing so, by means of a previously described technique of optical mapping. ${ }^{\text {s }}$

\section{Methods}

In this study we examined six normal men (aged 21-51 years) and 10 tetraplegic men (aged 16-71 years). All were studied in the same way, lying supine and breathing quietly or making slow vital capacity manoeuvres. The excursions of the thoracoabdominal wall were recorded by optical mapping. Briefly, the subject was photographed from above while a line pattern was projected on to his body from either side. The distortion of the line pattern by the surface of the body produces contour lines when viewed from an angle other than that of

Address for reprint requests: Dr MDL Morgan, Chest Medicine Department, East Birmingham Hospital, Birmingham B9 5ST.

Accepted 19 February 1985 projection. Because the camera-projector relationships were known, the three dimensional coordinates of the body surface could be extracted from the photograph. Some 500-1000 coordinates of the trunk surface were needed to generate adequate cross sections or compute the volume of those parts visible to the camera. Serial photographs were used to measure chest wall motion and respired volume. A continuous record of thoracoabdominal motion was often obtained at the same time with a video camera. In this case one typical quiet breath and one typical vital capacity measurement were chosen for analysis from the continuous record. The photographic records of these breaths were digitised manually, a process that takes $30-40$ minutes, so that they could be examined further.

To determine the abdominal and rib cage components of a breath the costal margin must be visible. Usually it could be seen with ease. When it could not, its end inspiratory and end expiratory positions were marked on the skin. It is important to mark both because there may be considerable sliding of the skin over the rib cage in deep breathing (fig 1).

The computer program for this study performs four principal steps: (1) The spatial $(x, y, z)$ coordinates of the 500-1000 points on the trunk surface are used to construct a digital image of the surface relative to a horizontal (that is, coronal) reference plane close to the bed. (2) The position of the costal margin on that surface is identified. (3) A vertical 

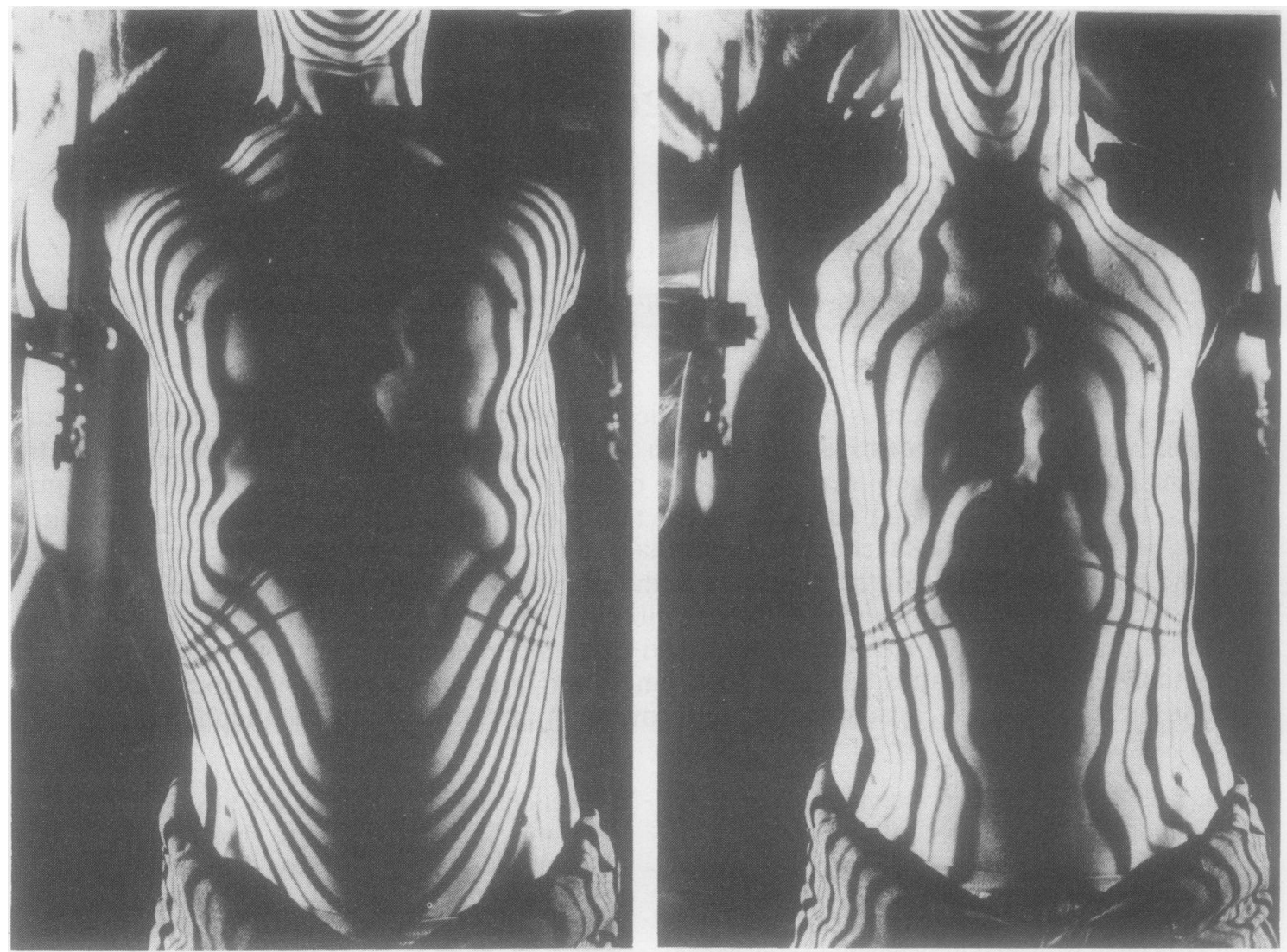

Fig 1 Torso with contour lines defining its shape; the three dimensional coordinates can be extracted to measure volume (left-inspiration; right - expiration). The costal margin can usually be identified easily on thin subjects, but if it cannot be seen the boundary must be marked with a felt pen.

boundary is dropped from that margin to divide the trunk image into rib cage and abdominal portions. (4) The instantaneous volumes and a selection of cross sectional (that is, transverse) profiles are generated for each portion.

\section{SUBJECTS}

The tidal volumes and vital capacity manoeuvres were photographed in runs of three breaths and the timing of the exposure was judged by eye. The breaths chosen for analysis were judged to be representative of the manoeuvre by observing the pattern change by eye on the still photographs and the video. After digitisation of the films from the selected breaths, the tidal volume and the vital capacity were calculated from the differences in trunk volume between inspiration and expiration. The measurement of respired volume in this way has previously been shown to give acceptable accuracy in both normal and tetraplegic subjects. ${ }^{5}$

Photographs of six normal male subjects were taken at the extremes of tidal breathing and of slow vital capacity manoeuvres. The distribution of ventilation was also determined in 10 patients with complete traumatic tetraplegia. Their level of injury was C5 or C6 as determined by clinical examination, and the duration of injury ranged from two weeks to 25 years. None of the patients had any respiratory complications at the time of study. In addition, one patient was studied on several occasions to record the change in pattern of ventilation with duration of injury. He was 18 years old at the time of his accident, when he sustained a transection of the spinal cord at the level of C6. There were no other injuries, the chest radiograph on admission was normal, and he had no subsequent respiration complications. He was studied on six occasions, $6,13,27,41,75$, and 272 days after injury. 


\section{Results}

NORMAL SUBJECTS

The findings for a typical quiet breath and a typical vital capacity manoeuvre in normal subjects are summarised in figure 2 , in which the respiratory excursions are expressed as a percentage of each subject's predicted vital capacity. ${ }^{6}$ In all six subjects the abdominal and rib cage excursions were outward-that is, positive-so that the total breath is the sum of the two components. The rib cage component varied from $5 \%$ to $56 \%$ (mean (SD) $35 \%(17 \%)$ ) during quiet breathing and from $21 \%$ to $68 \%$ (mean (SD) $40 \%(15 \%)$ ) over the vital capacity.

\section{TETRAPLEGIC SUBJECTS}

The findings in the 10 patients with tetraplegia are shown in figure 3 and follow the same convention.
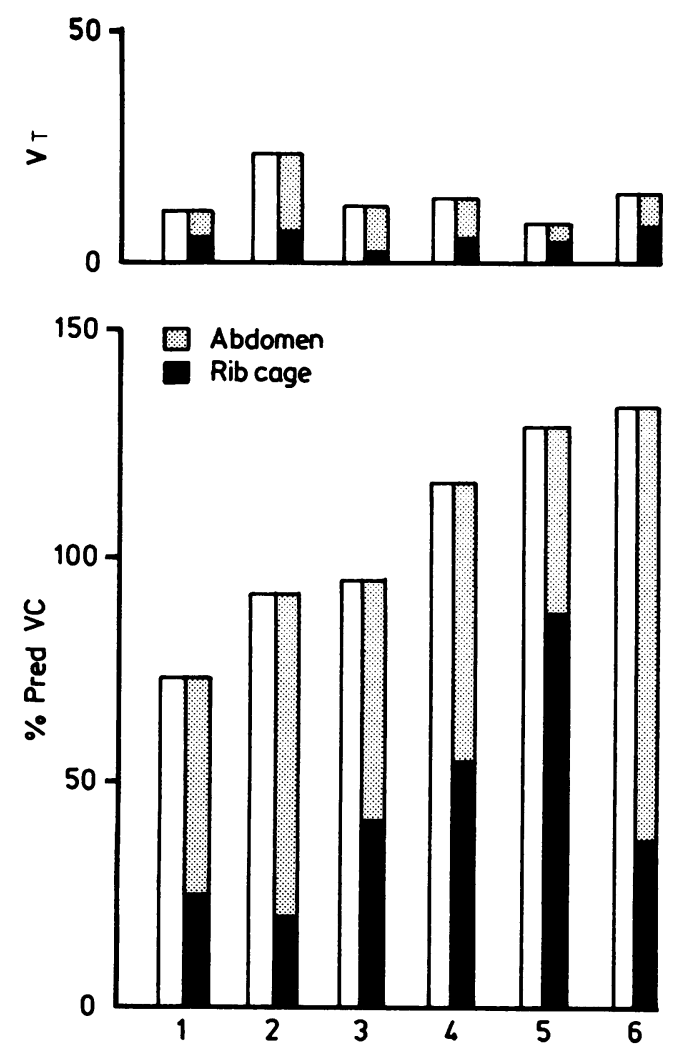

Fig 2 Tidal volume (VT) and vital capacity $(V C)$ of all the normal subjects, each expressed as percentage of predicted $V C$. The unshaded bars represent vital capacity or tidal volume and the shaded and black bars alongside show the abdominal and rib cage components of the excursion, which were in all cases positive. The abdominal contribution predominated in this position, accounting for $65 \%$ and $60 \%$ in the respective breaths.
The vital capacities range from $18 \%$ to $67 \%$ of predicted values for healthy people of same age, height, and sex. During quiet breathing the abdominal excursions were positive in all 10 subjects. The rib cage components were paradoxical (ingoing or negative) in six of the 10. In two patients ( 1 and 5) the rib cage contribution to tidal breathing predominated. Interestingly, both of these patients had abnormalities of the bony rib cage (severe pectus excavatum and ankylosing spondylitis). In the vital capacity manoeuvres the rib cage components became positive in three of the patients with paradox during tidal breathing but it remained negative in the other three.

The series of measurements made in one patient over the first nine months after injury are shown in figure 4 . In tidal breathing the paradoxical rib cage motion diminished and then reversed with time, and during the vital capacity manoeuvre rib cage motion was consistently orthodox and increased with time
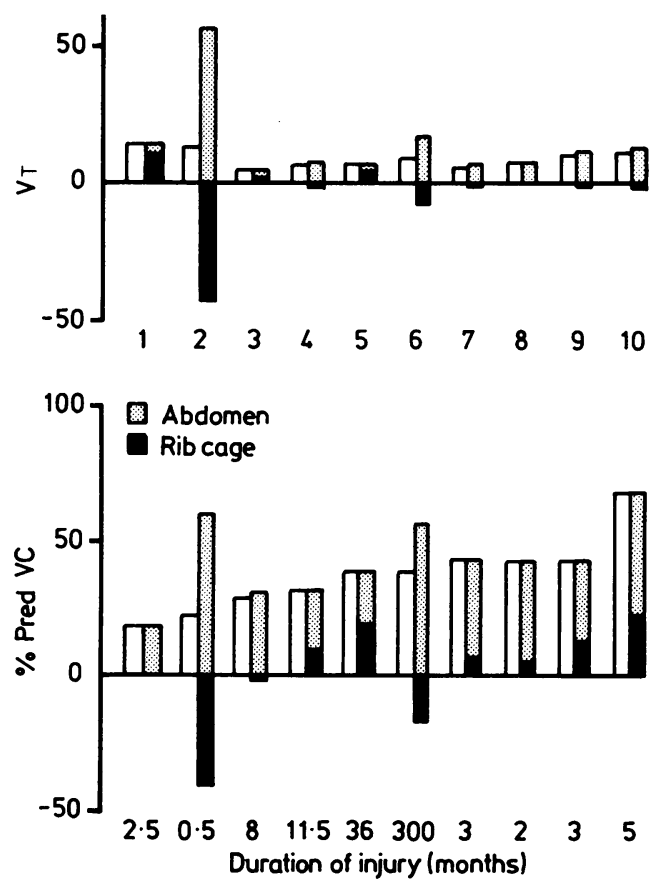

Fig 3 Partitioning of tidal breathing and vital capacity between the rib cage and the abdomen in 10 tetraplegic subjects. During tidal breathing $(V T)$ there was a negative rib cage contribution in six subjects. The largest volumes of paradox were seen in the two patients with the shortest and longest duration of injury. During the vital capacity (VC) manoeuvre many patients were able to reverse the negative rib cage contribution, but the two with the most severe degrees of tidal paradox were unable to do so. 
after the injury. Over this period the absolute contribution of the abdomen to the vital capacity more than doubled ( 715 to $1778 \mathrm{ml}$ ).

\section{Discussion}

Previous methods of determining the volume contributions of the rib cage and abdomen are of two types: one is partial plethysmography, developed by Bergofsky, where the lower part of the body is enclosed in a tank and the abdomen and rib cage are separated by a rubber seal around the costal margin. ${ }^{7}$ This method measures true volume change but is open to error because the position of the seal will change during a breath and cannot account for caudocranial movements of the rib cage. The other type of method uses measurements of circumference ${ }^{8}$ anteroposterior diameter, ${ }^{9}$ or cross sectional are $^{10}$ to derive volume change. In these methods the derivation depends on a relationship between the measured variable and the volume change, from which volume-motion coefficients can be calculated. For this the subject usually has to perform a calibration manoeuvre, which may only be valid in one posture. These methods also assume that a given
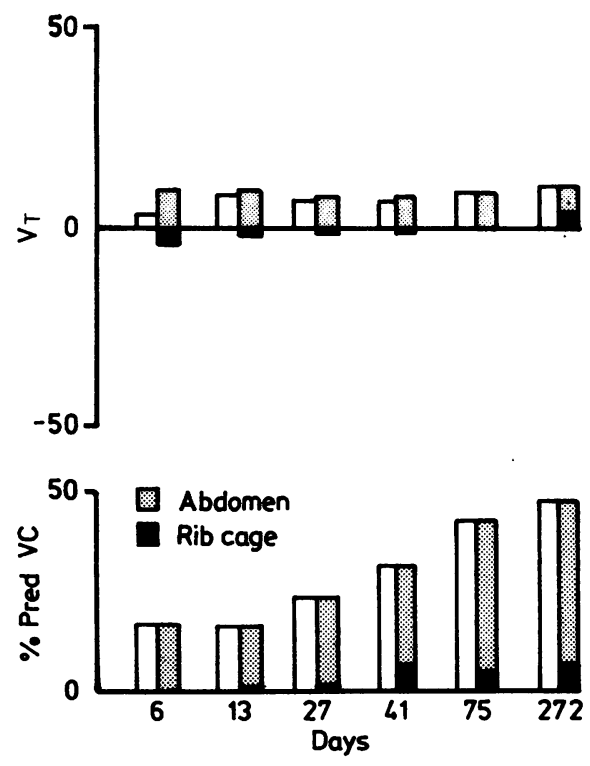

Fig 4 Results obtained in a single tetraplegic patient studied on six occasion after injury; the initial rib cage paradox during tidal breathing gradually diminished with time and then reversed. During the vital capacity manoeuvre there was no rib cage paradox and both the rib cage and the abdominal contribution increased with time. In this individual there was improvement in lung function with time without obvious neuromuscular recovery. dimension always implies a particular rib cage or abdominal volume when the airway is closed. Optical mapping makes no assumptions about shape and does not need to be calibrated for the individual. The partitioning is performed after the breath has been taken and along a boundary that can be clearly defined. Although the diaphragm itself is not visible the abdominal contents can be considered to be incompressible and therefore caudal displacement of the diaphragm will produce abdominal expansion of the anterior wall that is visible to the camera. Thus the increase in abdominal volume that is observed represents the volume displaced by the descent of the diaphragm.

The results of partitioning of ventilation in normal supine subjects by optical mapping are similar to those achieved by other methods." The rib cage and abdomen normally operate in parallel and the sum of their individual volume displacements makes up the total volume change of the respiratory system. In the normal subject these contributions are always positive.

Fugl-Meyer and Grimby ${ }^{12}$ attempted to partition ventilation in tetraplegia by using the method of Konno and Mead. They failed because the instability of the rib cage in tetraplegia invalidated the method. They did, however, report partial success in quiet breathing, where they described abdominal predominance. Earlier Bergofsky, using trunk plethysmography, which was much criticised at the time, obtained similar results to those described here. $^{13} \mathrm{He}$ found that abdominal expansion accounted for the major part of both tidal and exaggerated breathing and that in some patients there was rib cage paradox. In some of his patients the paradox was corrected on deep inspiration, while in others it increased. The results for patients 1 and 2 (see bottom of fig 3 ) illustrate an important point. They show that patients with very similar vital capacities and identical levels of injury may have a fourfold difference in displacement of the total chest wall. This in turn suggests that monitoring a tetraplegic patient's respiratory ability on the basis of vital capacity alone may be misleading.

The degree of motion of the rib cage in tetraplegic patients with similar levels of injury shows considerable individual variation. The ability of the rib cage to withstand the tendency to paradox appears to be determined by the degree of stiffness of the bony rib cage and possibly by the action of the neck accessory muscles. ${ }^{14}$ The duration of injury and the presence of intercostal muscle spasticity are not important factors in explaining the differences in rib cage motion between patients. In the patient who was studied sequentially after injury, however, there was an improvement in tidal volume and vital capacity 
that was not accompanied by any other clinical evidence of neuromuscular recovery. One obvious explanation for this would be progressive improvement in diaphragmatic function after the resolution of oedema of the cord soon after injury. While this is known to occur in some cases, this patient was screened fluoroscopically one week after injury, when the diaphragm appeared to move well. The increase in the abdominal component may therefore have a different explanation-for example, stiffening of the rib cage through joint stiffness, or perhaps even intercostal spasticity, and atrophy of the abdominal wall allowing the diaphragm to move more effectively than before.

Within individuals therefore function appears to improve with time after injury. Thus rib cage paradox may be only a temporary phenomenon in some patients while in others it may improve but never resolve.

\section{References}

1 Mortola JP, Sant' Ambrogio G. Motion of the rib cage and the abdomen in tetraplegic subjects. Clin Sci Mol Med 1978;54:25-32.

2 Fugl-Meyer AR, Grimby G. Ventilatory function in tetraplegic patients. Scand J Rehabil Med 1971; 3:151-60.

3 Moulton A, Silver JR. Chest movements in patients with traumatic injuries of the cervical cord. Clin Sci 1970;39:407-22.
4 Ledsome JR, Sharp JM. Pulmonary function in acute cervical cord injury. Am Rev Respir Dis 1981; 124:41-4.

5 Morgan MDL, Gourlay AR, Denison DM. An optical method of studying the shape and movement of the thoracoabdominal wall in recumbent patients. Thorax 1984;39:101-6.

6 Cotes JE. Lung function: principles and application in medicine. 3rd ed. Oxford: Blackwell Scientific Publications, 1975.

7 Bergofsky EH. Relative contributions of the rib cage and diaphragm to ventilation in man. J Appl Physiol 1964;19:698-706.

8 Agostoni E, Mognoni P, Torri G, Saracino F. Relation between changes of rib cage circumference and lung volume. J Appl Physiol 1965;20:1179-86.

9 Konno K, Mead J. Measurement of separate volume changes of rib cage and abdomen during breathing. $J$ Appl Physiol 1967;22:407-22.

10 Sackner JD, Nixon AJ, Davis B, Atkins N, Sackner MA. Non-invasive measurement of ventilation during exercise using a respiratory inductive plethysmograph. Am Rev Respir Dis 1980;122:867-71.

11 Sharp JT, Goldberg NB, Druz WS, Danon J. Relative contributions of ribcage and abdomen to breathing in normal subjects. J Appl Physiol 1975;39:608-18.

12 Fugl-Meyer A, Grimby G. Rib cage and abdominal volume ventilation partitioning in tetraplegic patients. Scand J Rehabil Med 1972;4:161-7.

13 Bergofsky EH. Mechanism for respiratory insufficiency after cervical cord injury. Ann Intern Med 1964;61:435-47.

14 Morgan MDL, De Troyer A. The individuality of chest wall motion in tetraplegia. Clin Respir Physiol 1984; 20:547-52. 